\title{
A Full 3D Finite Element Model for Buckling Analysis of Stiffened Steel Liners in Hydroelectric Pressure Tunnels
}

J.L.G. Valdeolivas, J.C. Mosquera

\begin{abstract}
The availability of tools for safety evaluation of a pressure liner is a relevant issue in both Structural and Hydraulic Engineering. A numerical procedure for assessing the stability of a stiffened steel liner in a hydroelectric pressure tunnel is presented in this paper.

First, a review of some analysis methods for steel liners is outlined. Relevant aspects for the critical buckling pressure assessment are considered, specifically boundary conditions and geometric imperfections.
\end{abstract}

General 2D and 3D nonlinear finite element modeling procedures, including large displacements formulation are presented. Some relevant factors in the liner response, such as the annular gap, the stiffeners and the interaction with the elastic medium surrounding the steel liner are taken into account.

As a result, some simple modeling guidelines for thin-walled steel pressure liners are depicted. Also, some conclusions regarding the influence of stiffeners as well as of the surrounding medium are drawn.

The procedure is applied to an actual steel tunnel liner which failed by global instability in 2010 in Spain. The aim is to simulate the causes of collapse as well as to draw some design criteria.

Key Words: buckling, steel liner, pressure tunnel, finite element method, hydroelectric power plant 


\section{Introduction}

The stability of hydropower pressure tunnels has been a field of interest for researchers since the decade of 60 's. The increase in wind power generation and the need to introduce regulation to the grid have led to the development of new large-scale hydroelectric projects, such as reversible or pump-storage schemes. These involve high flows and heads, which entail large diameters for hydraulic pressure tunnels.

In addition, it has become necessary to check the safety of existing or rehabilitated hydroelectric power plant penstocks, some of them with more than 50 years of operation [1].

More recently, large pump-storage projects require high-strength steel liners, frequently subjected to pulsating action mainly due to secondary regulation.

External pressure is often the most demanding loading for the analysis of a steel liner encased in a pressure tunnel, which may fail by buckling or instability.

A variety of buckling solutions for cylindrical shells under different conditions are described in the literature. There are solutions available for unconstrained cylindrical shells subjected to various types of loading (used in other Engineering fields, i.e. buried pipes, submarine and offshore structures, aircraft structures, ...) $[2,3,4,5]$, as well as for unconstrained cylindrical shells, either with or without stiffeners, related to general topics of instability of shells $[6,7,8,9,10]$.

Buckling failure occurs when most of the strain energy, stored as membrane energy, is transformed into bending energy, involving large deformations and deflections, which may result in catastrophic and brittle failure. Another type of failure may occur when the steel elastic yield stress is reached, which may lead to inelastic buckling [11].

Some practical measures to prevent buckling have usually been increasing either the liner wall-thickness or the shell rigidity by attaching stiffening elements.

Thin-walled unstiffened cylindrical shells, with low buckling resistance, are prone to collapse by non-symmetric bifurcation buckling (multiple-lobe mode in a free cylinder, and either single-lobe or two-lobe modes for the constrained cylinder case). However, for stiffened cylinders, both the thinwalled shell and the stiffeners can altogether collapse due to external pressure as well. Such type of failure is known as general instability. Otherwise, if stiffeners are strong enough, the thin shell span between stiffeners may collapse, which is usually said to be local collapse. 
The type of buckling mode for stiffened liners (i.e., global instability or local collapse) cannot be identified beforehand. Rather, the failure mode must be inferred from the ratio of the stiffness of the thin-walled cylindrical shell to that of the stiffeners (i.e. their shape and spacing).

The numerical procedure hereby described is applied to the analysis of an actual steel liner encased in a pressure tunnel of a hydroelectric power plant located in Northern Spain. During summer of 2010, the stiffened steel liner buckled under external pressure. In order to find the collapse causes and to achieve design rules as well, a finite element simulation procedure has been developed.

\section{Review of some buckling theoretical approaches}

The buckling of an unsupported circular ring subjected to external pressure was first studied by Donnel (1956) derived the elastic buckling pressure value for cylindrical shells under external pressure $[9,12]$ :

$$
P c r=\frac{\pi^{2} E}{12\left(1-v^{2}\right)}\left(\frac{t}{l}\right)^{2}\left\lfloor\frac{\left(1+m^{2}\right)^{2}}{m^{2}}+\frac{12 Z^{2}}{\pi^{4} m^{2}\left(1+m^{2}\right)^{2}}\right\rfloor
$$

Where:

$E$ is Young's Modulus;

$t$ is the shell thickness;

$v$ is Poisson's ratio;

I is either the spacing of stiffeners or the length between radially constrained crosssections;

$m=n l / \pi R$

$n$ is the number of lobes appearing in the buckling mode;

$R$ is the radius of the cylindrical shell;

$Z$ stands for the Batdorf parameter: $Z=\frac{l}{R} \sqrt{1-v^{2}}$

Timoshenko [7] derived formulations for unconstrained shells (elastic buckling). Other authors $[6,8,9]$ have stated either general or specific solutions for the buckling problem of unconstrained cylindrical shells. Vaughan [13] and Borot $[14,15]$ developed formulations for multiple-lobe buckling in the particular case of constrained shells. 
Glock (1977) applied a nonlinear deformation theory for analyzing the stability of elastic rings encased in a rigid medium under external pressure $[10,16]$.

Glock assumed two deformation regions for the elastic ring: a buckled part (in which there is no contact between the ring and the external concrete) and an unbuckled zone, which is in contact with the rigid host pipe. The approximate radial deflection for the former is given by:

$$
\omega(\theta)=\delta \cos ^{2}\left(\frac{\pi \theta}{2 \phi}\right)
$$

where

$\phi$ is the angle defining the border between the buckled and unbuckled regions,

$\delta$ is the amplitude of the buckled shape and

$\theta$ is the angular coordinate.

The total potential energy of the system is given by:

$\mathrm{II}=U_{1}+U_{2}-W$

where

$W$ is the work potential of external hydrostatic pressure,

$U_{1}$ and $U_{2}$ are, respectively, the strain energy in the buckled and unbuckled portions of the ring.

By assuming a constant hoop axial force around the ring, $N$, the application of the principle of minimum total potential energy with respect to both $\delta$ and $\phi$, leads to expressions for pressure $p$, for $\delta$ and for $N$ with respect to parameter $\phi$. Finally, minimization of pressure in terms of $\phi$ yields the following closed-form expressions for the critical pressure, the corresponding angle $\phi$ and the corresponding amplitude of the buckled shape:

$\frac{P_{c r} R^{3}}{E I}=0.969\left(\frac{E A R^{2}}{E I}\right)^{2 / 5}$

$\left(\frac{\pi}{\phi}\right)_{c r}=0.856\left(\frac{E A R^{2}}{E I}\right)^{1 / 5}$

$\left(\frac{\delta}{R}\right)_{c r}=2.819\left(\frac{E I}{E A R^{2}}\right)^{2 / 5}$

$E I$ and $E A$ are, respectively, the flexural and the axial stiffnesses. 
By assuming the plane-strain condition of long pipes, that $E$ is constant for both flexural and axial stiffness, and a thin-walled ring encased in a rigid cavity, the following expression for the elastic buckling pressure was obtained:

$$
P_{c r}=\frac{E}{1-v^{2}}\left(\frac{t}{D}\right)^{2.2}
$$

Comparisons with experimental data, as well as with nonlinear finite-element results $[10,11]$ have shown that Glock's formula can predict quite accurately the buckling pressure of tightly fitted elastic cylinders in a rigid cavity.

Jacobsen $[17,18]$ derived equations to calculate the inelastic buckling pressure of loosely fitted liners. He only considered the effect of normal stresses in the liner's cross section on its yielding and ignored the effect of longitudinal stress (i.e., plane-stress assumption)

Boot [19] extended Glock's solution by considering the effect of a gap, and achieved the following semi-analytical solution for elastic buckling:

$P_{c r}=\frac{E^{\prime}}{12} \gamma^{2}\left(\frac{t}{R}\right)^{3}\left\lfloor 1+\frac{1}{6} \sqrt{\left[16-\frac{20}{9}\left(\frac{\mathrm{t}}{\mathrm{R}}\right)^{2} \gamma^{5}-6 \frac{\delta}{\mathrm{R}} \gamma^{3}\right]}\right]$

where $\delta$ is the value of annular gap and $\gamma$ is given for the formula:

$\left[2 \sqrt{\left[16-\frac{20}{9}\left(\frac{\mathrm{t}}{\mathrm{R}}\right)^{2} \gamma^{5}-6 \frac{\delta}{\mathrm{R}} \gamma^{3}\right]}+\frac{1}{3}\left[16-\frac{20}{9}\left(\frac{\mathrm{t}}{\mathrm{R}}\right)^{2} \gamma^{5}-6 \frac{\delta}{\mathrm{R}} \gamma^{3}\right]-\frac{1}{12}\left[\frac{100}{9}\left(\frac{\mathrm{t}}{\mathrm{R}}\right)^{2} \gamma^{5}+18 \frac{\delta}{\mathrm{R}} \gamma^{3}\right]\right\rfloor=0$

Based on results from FE models, El-Sawy [20] proposed the following regression solution for elastic buckling:

$$
P_{c r}=\frac{E^{\prime}}{4}\left(\frac{t}{R}\right)^{3}\left\lfloor\frac{25+350 \frac{t}{R}+315 \frac{\delta}{R}}{0,15+65 \frac{t}{R}+350\left(\frac{t}{R}\right)^{2}+145 \frac{\delta}{R}}\right\rfloor
$$

where $\delta$ is the maximum size of the annular gap between the liner and the host pipeline.

El Sawy et al [21] studied the effect of local wavy imperfections on the elastic stability of cylindrical liners by using a 3D finite-element model, and derived empirical formulae that agree with Glock's model. 
Thépot (2000) derived a series of critical values when a liner buckles. He achieved the following formula for a circular liner with a single lobe in the elastic range [22]:

$$
\frac{P_{c r}}{E^{\prime}}=\frac{0.218}{1+0.41 \beta-0.006 \beta^{2}}\left(\frac{t}{R}\right)^{2.2}
$$

with $\beta$ being

$$
\beta=3^{0.6}\left(\frac{d}{R}\right)\left(\frac{R}{t}\right)^{1.2} \leq 10.0
$$

and $E^{\prime}=E /\left(1-v^{2}\right)$

In the above expressions, $E^{\prime}$ is the equivalent Young's modulus under plane-strain assumption, $t$ is the steel thickness, $R$ is the liner radius, and $L$ is the spacing between stiffeners.

Vasilikis and Karamanos [11] developed a plastic hinge mechanism and achieved a closed-form solution to illustrate the post-buckling of the liner. They also defined a slenderness parameter $\lambda$, which shows that buckling occurs in the inelastic range when $D / t$ ranges from 100 up to 400 (usual values for hydroelectric power plants).

Buckling analysis of steel cylindrical liners in hydraulic pressure tunnels and penstocks can be grouped into the following approaches $[15,23,24]$ :

- Multiple-lobe mode buckling: A cylindrical thin shell, in which radial displacements are unrestrained, is subjected to uniform external pressure. Depending on its geometry, on its boundary conditions and on the external pressure, the symmetric buckled shape of the steel liner is likely to include two or more lobes. These approaches are useful for obtaining the buckling pressure of the liner span between two consecutive stiffeners and the overall buckling pressure in aerial pipes as well.

- Radial symmetry buckling: the liner is considered to be encased in the surrounding medium. Hence, there is a radial confinement provided by either the concrete pipeline or the rock. The deformed layout of the liner is assumed to include two or more lobes symmetrically. The annular gap is assumed constant.

- Single-lobe buckling mode: it is assumed that the liner and the cavity are in contact at a discrete set of points, having lost the initial uniform annular gap. In case the external pressure reaches a critical value, the cylinder buckles under single-lobe mode. 
There are two limits (i.e. lower and upper ones) which define the actual buckling pressure value, depending on the approach followed: the former can be obtained from the linear elastic buckling theory (unconstrained cylinder). Instead, Glock's formulae [16] lead to an upper limit value (fully constrained cylinder).

As a whole, a large number of approaches, including Glock's equation for fully constrained pipes, lead to expressions with the same general pattern:

$$
P_{c r}=c E^{\prime}\left(\frac{t}{D}\right)^{m}
$$

where $c$ and $m$ are constants, so that:

- $\quad c=2, m=3$ for an unconstrained cylinder (elastic buckling) [7];

- $\quad c=1, m=2.2$ for Glock's fully constrained cylinder $[10,16]$.

Winderburg and Trilling's equation (1934) is useful for determining the number of waves, $n$, in the complete circumference of the steel liner at collapse [25]:

$$
n=\sqrt[4]{\frac{6 \pi^{2}}{\left(\frac{L}{R}\right)^{2} \frac{t}{R} \sqrt{1-v^{2}}}}
$$

\section{Finite Element model for buckling analysis of a thin-walled steel liner}

First, a simple 2D geometrically nonlinear plane-strain finite element model is implemented for assessing the buckling response of a steel liner encased in a hydroelectric pressure tunnel subjected to external pressure.

Also, some full 3D models were implemented in order to account for specific issues such as stiffeners and boundary conditions. Four-node quadrilateral shell elements with six degrees of freedom per node have been used for 3D modeling of the thin-walled cylindrical liner (Figure 1). The formulation applied combines membrane and plate-bending behavior. Transverse shear effect has been neglected (i.e., thin shell theory is applied). It has been assumed that there is no friction between the steel liner and the surrounding concrete. Vasilikis and Karamanos [11] in demonstrates that in very soft confinement medium, the consideration of friction results in a small increase of the ultimate capacity and in the case of rigid confinement, this effect has been found to be negligible. Beam-type elements have been used for modeling the stiffeners. 


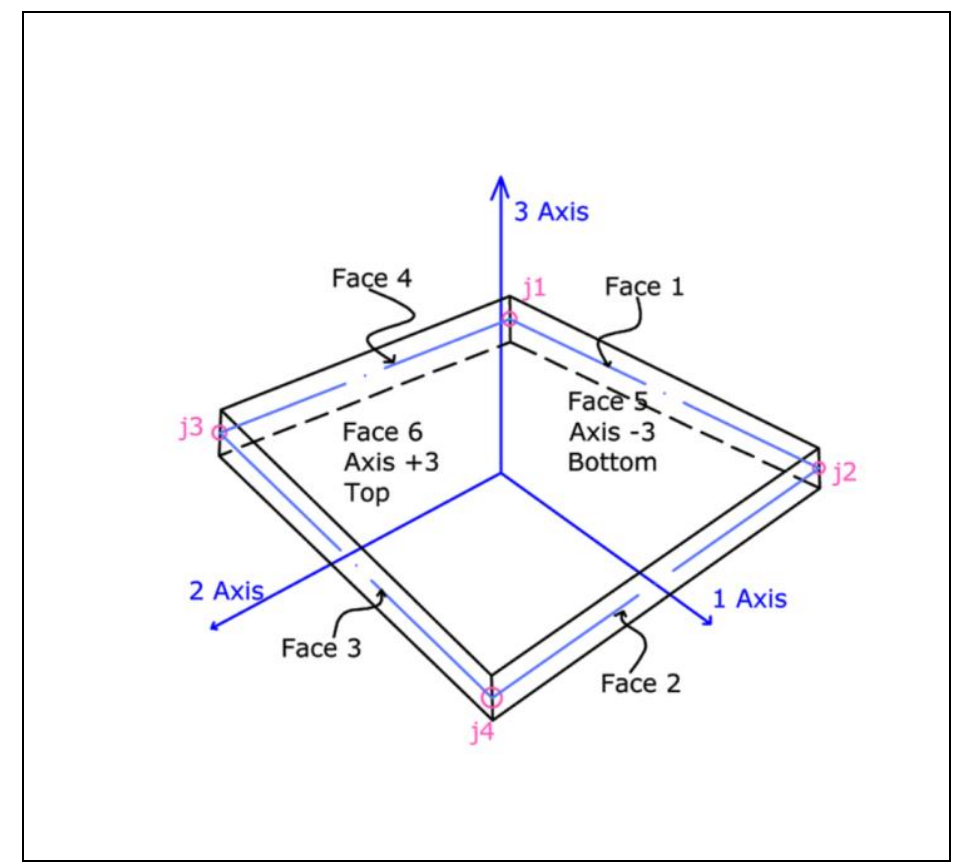

Figure 1. Four-node finite shell element

\subsection{Linear buckling vs nonlinear buckling analysis}

The linear buckling analysis involves the solution of the eigenvalue problem:

$[K-\lambda G(f)] \psi=0$

where

$K$ is the stiffness matrix of the structure,

$\lambda$ is the matrix of buckling factors,

$G(f)$ is the geometric stiffness matrix due to the load vector $f$,

$\Psi$ is the matrix of corresponding eigenvectors.

Linear buckling theory gives an assessment of the critical load of an ideally elastic structure. This analysis yields the buckling modes and the safety factor against instability. Neither geometrical imperfections nor non-linear behavior (material, geometric, boundary conditions ...) can be considered in this type of analysis [26]. For this reason, linear buckling has been used to determine the buckling pressure and the corresponding mode for unconstrained liners, which may be idealized as cylindrical shells. 
Negative values of buckling factors mean that buckling occurs if loads are reversed [27]. The behavior of cylindrical shells under external pressure has been found to be very sensitive to geometric imperfections. The major factors affecting the bifurcation pressure are the diameter-tothickness ratio $D / t$, Young's modulus $E$, and initial imperfections such as ovality, non-constant wall thickness, eccentricity (non-uniform annular gap) as well as the presence of welding joints. When nonlinear buckling analysis is performed, equilibrium equations are posed for the deformed configuration of the structure. An initial imperfection is necessary in order to trigger the buckling process.

Both geometric and material nonlinearities can be considered. This includes both P-Delta effects and large displacements formulation. The former can be properly applied for buckling analysis, especially for snap-through buckling and post-buckling behavior whereas the latter is applicable for inelastic buckling analysis.

General purpose nonlinear finite-element program SAP2000 [27], used in the numerical study, provides two suitable methods for this type of simulations, i.e. Nonlinear Static Analysis and Nonlinear Time-History Analysis. The latter yields better and more accurate results. Hence, the dynamic response of a structure subjected to a specified time-varying loading is obtained through a step-by-step direct integration technique. However, the computer effort is larger than when applying modal analysis.

Moreover, both direct-integration results and convergence are highly sensitive to time-step size. For this reason, the direct-integration time-history analysis seems to be computationally more efficient, leading to accurate and realistic solutions, rather than nonlinear static analysis does. Besides, by applying the former, both the structural stability and stress conditions may be checked at each time step.

Nonlinear buckling analysis may be also carried out by performing a nonlinear incremental stepby-step stress analysis, by checking, as load increases at each step, the stress level and the slope variation along the pressure-deflection curve (Figure 12).

External pressure is applied incrementally. A variety of common methods are available for performing nonlinear buckling analysis. The "Hilbert-Hughes-Taylor alpha" (HHT) method, with alpha taking values between 0 and -0.33 , is used.

Since direct integration results are extremely sensitive to time-step size [27], the selection of appropriate values for time integration parameters has been found to be relevant. 


\subsection{The surrounding boundary and ground-system interaction.}

Interaction between the steel liner and the surrounding concrete and rock must be properly simulated in order to assess the system response.

An initial non-uniform annular gap is considered in all constrained models developed.

Nonlinear support conditions may arise during system deformations. Thus, a nonlinear contacttype element is used in order to account for the ground-system interaction. Since friction is disregarded, a 1D-gap finite element is implemented in the static model. This link element is assumed to withstand only compression forces. The key parameters for defining its behavior are the initial annular gap amplitude $g 0$ and the axial stiffness $k$, so that, for an initially given $g 0$ (Figure 2):

- $k=E_{r} / R$ [22], when $g a p<g 0$, with $E_{r}$ being the Young's modulus of the rock or backfill and $R$ is the radius of the penstock.

- $k=0$ if $g a p>g 0$.

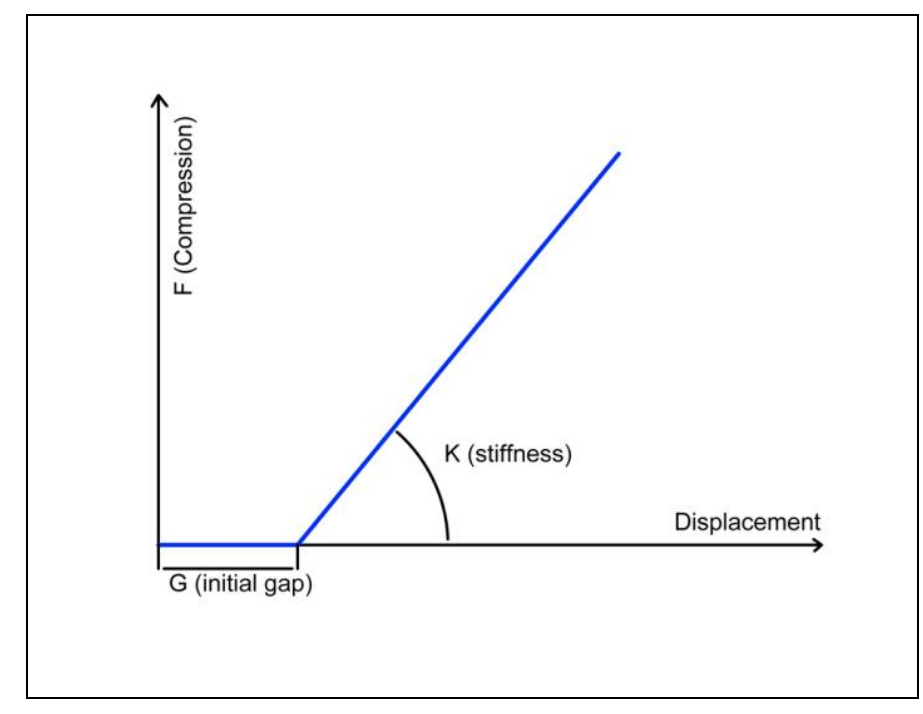

Figure 2. Gap contact-type element

The parameter $k$ accounts for the stiffness of the liner backfill and thus prevents the liner from free deformation. It has a significant effect on the model response, i.e, on the buckled shape mode $(n=2,3,4 \ldots)$ and on the critical external pressure as well.

As regards the stiffeners, two types of behaviors are pointed out:

- Stiffener: Only provides the cylindrical shell with rigidity, but does not restrain radial displacements. They are usually applied in aerial pipes. 
- Connector: Provides stiffness to the thin-walled liner, mainly involving radial constraint, thus reducing the length of the free span of the shell. Welds between stiffeners and the shell should be designed accordingly and properly executed in order to withstand the external pressure.

\section{Case study: the collapse of an actual hydropower pressure tunnel}

The earlier procedure is applied to simulate the response of an actual concrete-encased steel liner in a pressure tunnel of a hydroelectric power plant in northern Spain. The steel liner collapsed by global buckling during summer of 2010 (Figure 3).

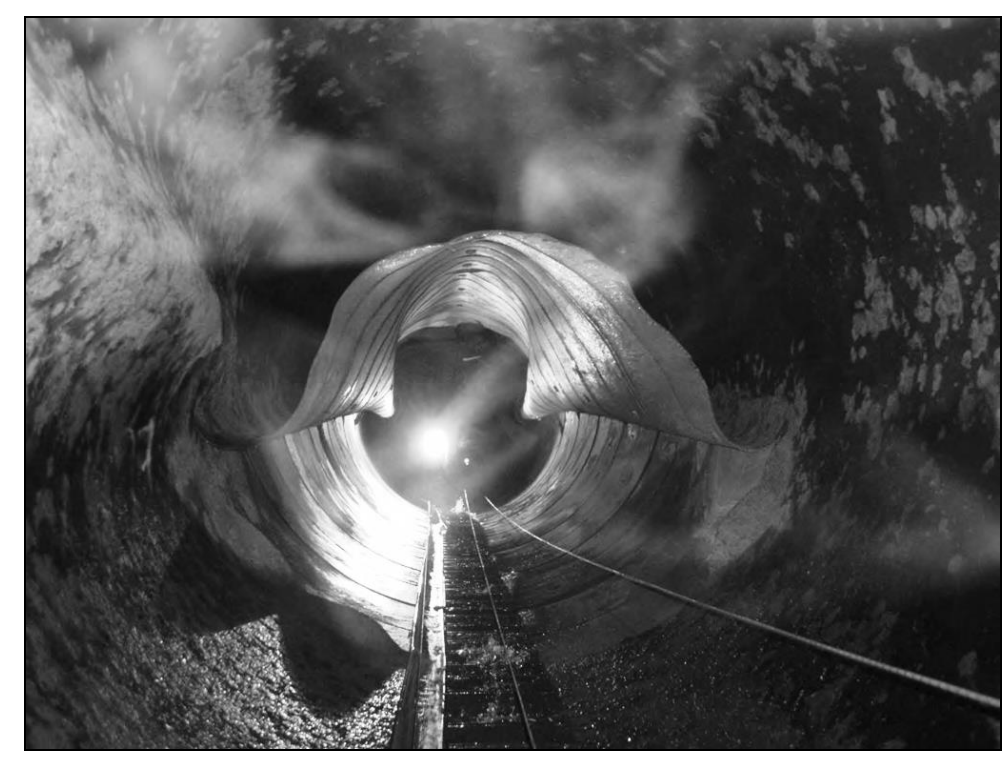

Figure 3. View of damaged steel liner pressure tunnel

The underground hydroelectric power plant is equipped with three groups, with a total capacity of $276 \mathrm{MW}$ and a maximum gross head of $135.7 \mathrm{~m}$. The design flow is $210 \mathrm{~m}^{3} / \mathrm{s}$.

The steel liner is a thin-walled cylindrical shell with variable wall thickness ranging from $14 \mathrm{~mm}$ up to $32 \mathrm{~mm}$ as penstock depth increases. The inner diameter is $4.0 \mathrm{~m}$; the outer concrete pipeline is around $0.75 \mathrm{~m}$ thick, so that the excavation average diameter is $5.5 \mathrm{~m}$.

The steel liner was not anchored to the concrete host pipeline, although was built up with $0.1 \mathrm{~m}$ high triangular-shaped stiffeners embedded in concrete. The spacing ofm stiffeners is $2.5 \mathrm{~m}$. The total length of the pressure tunnel is 160 meters. The intake horizontal section is about 7 meter long, ending with an elbow. It is followed downstream by a 108 meter long shaft inclined 
$40^{\circ}$ and a 52 meter long horizontal section, connecting the pipeline to the spiral case in the underground power plant (Figure 4).

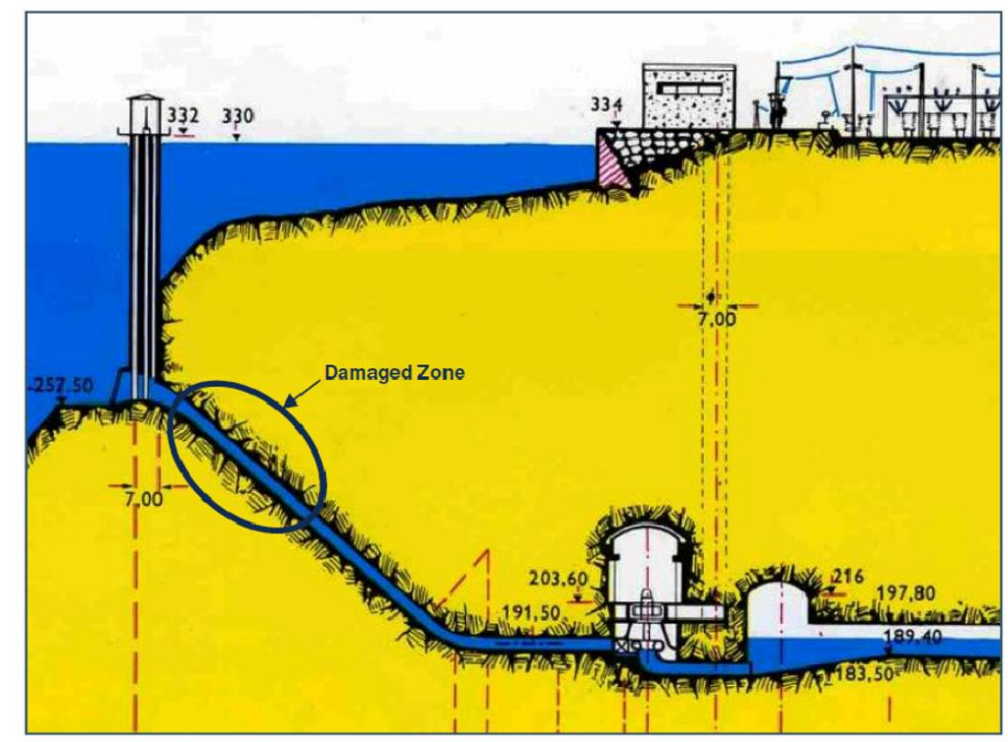

Figure 4. Hydroelectric pressure tunnel longitudinal profile of case study

A simulation of the structural behavior of the steel liner is performed. As pointed out in related literature, results obtained show that the critical buckling pressure of the liner is strongly dependent on the boundary conditions. This is to say, the type of support and degree of constraint, the presence and performance of stiffeners as well as eventual geometric imperfections have significant effect on the system stability. For this reason, different liner models, with and without stiffeners, are carried out for various types of confinement, gap amplitude and geometric imperfections.

As shown in table 1, current analytical methods cannot address accordingly all the issues associated with liner stability under external pressure. Hence, the finite element method is a powerful tool for analyzing such type of problems.

Under uniform external pressure assumption, both linear and nonlinear buckling analysis have been implemented in order to simulate the steel liner collapse. 


\begin{tabular}{|c|c|c|c|c|c|c|c|c|}
\hline ELEM ENT & \multicolumn{4}{|c|}{ STEEL LINER (Pressure Tunnels) } & \multicolumn{4}{|c|}{ STEEL LINER (Shaft) } \\
\hline Constrains & \multicolumn{4}{|c|}{ CONSTRAINED (Variable Gap) } & \multicolumn{4}{|c|}{ CONSTRAINED (Uniform Gap) } \\
\hline Type & \multicolumn{3}{|c|}{ Stiffened } & Unstiffened & \multicolumn{3}{|c|}{ Stiffened } & Unstiffened \\
\hline Buckling Type & \multicolumn{2}{|c|}{ Local Instability } & General Instability & Shell Buckling & \multicolumn{2}{|c|}{ Local Instability } & General Instability & Shell Bucklng \\
\hline Mode failure (frequently) & \multicolumn{2}{|c|}{ Multiple lobes } & One (or two) lobes & One ( or two) lobes & \multicolumn{2}{|c|}{ Multiple lobes } & Multiple lobes & Multiple lobes \\
\hline Border conditions & \multicolumn{2}{|c|}{ Stiffener } & Continuity & Continuity & \multicolumn{2}{|c|}{ Stiffener } & Continuity & Continuity \\
\hline Traiditional calculation & Radial symmetry & Multiple lobes & Jacobsen, Amstuz? & Single lobe & Radial symmetry & Multiple lobes & $?$ & Radial symmetry \\
\hline FEM calculation & \multicolumn{2}{|c|}{ Linear analisys ? / Nonlinear analysis } & Nonlinear analysis & Nonlinear analis & \multicolumn{2}{|c|}{ Linear analysis? / Nonlinear Analysis } & Nonlinear analysis & Nonlinear analysis \\
\hline
\end{tabular}

Table 1. Appropriate calculation methods depending on the type of pressure tunnel.

\subsection{Finite element model features}

The key features and assumptions made in the analysis of this case study are:

- Liner inner diameter: $D=4.0 \mathrm{~m}$.

- Liner length: 30m (i.e. edge effects at the mid-span cross-section can be neglected).

-Wall thickness: $t=14 \mathrm{~mm}$ for 2D and 3D models. Also, in a 2D parametric analysis, $t$ is varied from 10 up to $32 \mathrm{~mm}$, i.e. $D / t$ ratio ranging between 100 and 400 , which is typical for steel liners in hydropower penstocks.

- Steel assumed is elastic, perfectly plastic material. Yield strength is taken according to Von Mises' criterion. Besides:

- Young's modulus: $E=210 \mathrm{GPa}$.

- Yield stress of steel: $F_{y}=235 \mathrm{Mpa}$.

- Poisson's ratio: $v=0.3$

- Friction between the liner and the encasing concrete is disregarded.

- Estimated maximum external pressure: $P=0.70 \mathrm{MPa}$. This load is applied through a linearly incremental procedure; starting from zero up to the maximum value (could be greater than 0.7 Mpa).

- Eigenvalue convergence tolerance for linear buckling analysis: 1e-10

- Finite element model of a 2.5 meter long liner: 800 shell-type elements for the cylindrical thinwalled shell.

- Finite element model of a 30 meter long liner: 9600 shell-type elements for the thin-walled cylinder, involving 80 shell elements along the cross-section circumferential perimeter. When stiffeners are considered, 1040 beam-type elements are involved to account for their effect. 


\subsection{Nonlinear 2D analysis of a constrained steel liner}

This is the simplest analysis for the constrained cylinder case. The FEM model consists of an assemblage of 2D beam-type elements. Displacement constraints due to confinement are accounted for through link-type finite elements, which only withstand compression forces as functions of a predefined annular gap. Plane-strain conditions are assumed.

Results of 2D models have been successfully checked with available closed-form analytical solutions: Glock [16], El-Sawy [16,17], Thepot [22], Boot [19], Jacobsen [17,18], Montel [28], Li[24] and Prabu [29]. Hence, 2D models are used for calibrating the 3D numerical models implemented in this work.

A nonlinear direct-integration time-history analysis including P-Delta effects and large displacements is performed. External pressure is applied through a linearly increasing procedure. The following parameters have been used for P-Delta analysis:

- $\quad$ substep size between 0.01 and 1e-4;

- $\quad$ maximum number constant-stiff iterations per step: 15

- $\quad$ maximum number of Newton-Raphson iterations per step:15.

It is essential to apply some kind of imperfection to the model, which is a priori perfectly symmetric, in order to locate the buckling lobe [20]. Thus, a uniform $0.60 \mathrm{~mm}$ annular gap amplitude and a $0.10 \mathrm{~mm}$ maximum ovality (out of roundness) at the top of the cylinder are considered as initial imperfections.

Moreover, due to symmetry conditions, the model consists only of half of the cylinder, involving an assemblage of 40 beam-type elements and 40 distributed gap-type elements.

In order to account for the annular gap as well as for the flexibility of the surrounding medium, a simple contact-element is used. Its nonlinear behavior is described through a stiffness parameter, $k$ (with units $[F / L])$, so that $k=E_{r} \Omega / R[30]$

where

$E_{r}$ is Young's modulus of the surrounding medium.

$\Omega$ is the tributary area of each gap element.

$R$ is the liner radius. 
Some pressure-displacement curves corresponding to different values of the stiffness parameter $k$ of gap elements are shown in Figure 5. The effect of the stiffness of the surrounding elastic medium on the analysis results is also shown, i.e. two cases are pointed out:

1) For $k=0.8 \mathrm{kN} / \mathrm{m}$. In this case, the liner behaves as if it were a free cylinder (Euler buckling), i.e. the surrounding medium has negligible effect on the buckling pressure.

2) When the stiffness of the confinement ranges from $k=400$ to $40,000 \mathrm{kN} / \mathrm{m}$. In particular, the value $k=400 \mathrm{kN} / \mathrm{m}$ corresponds to the rigidity of a low-quality encasing concrete ( $E=13,000$ to $20,000 \mathrm{MPa})$.

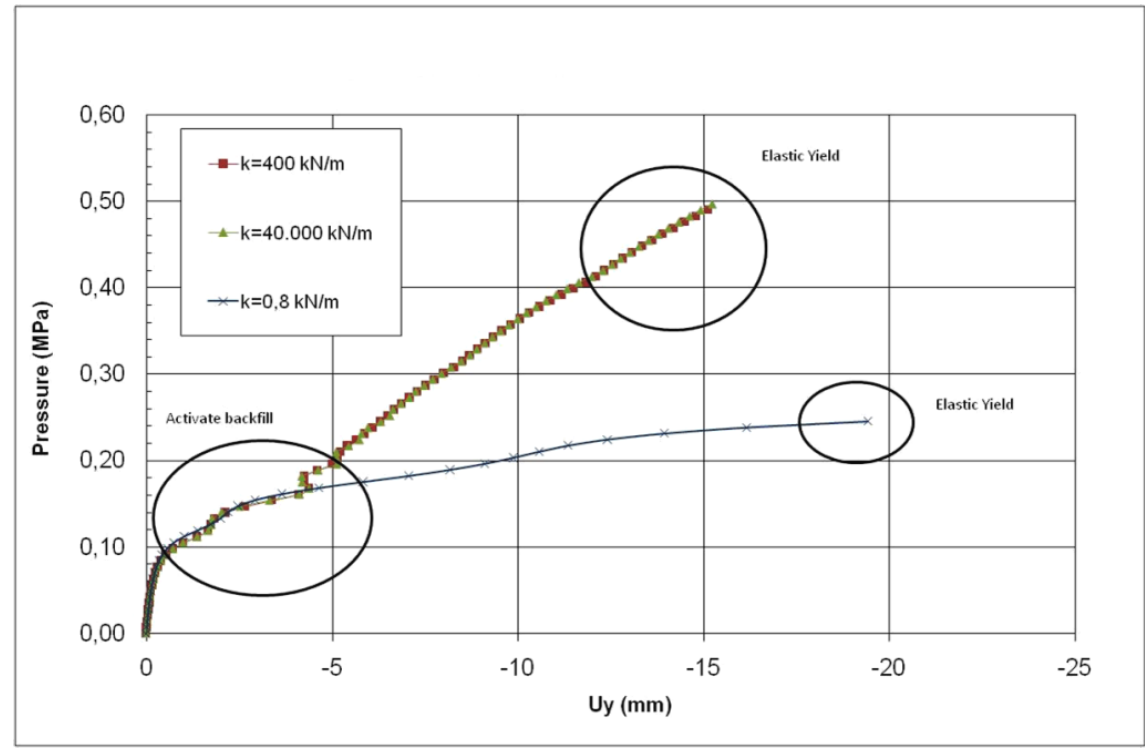

Figure 5. Pressure-displacement (radial displacement Uy in the node located at the top of the cylinder, where geometrical imperfection is applied) for different stiffness values of the backfill (k). D/t=285.7

Hence, the stiffnesses of both the concrete pipeline and the encasing rock are parameters not often considered in earlier studies. However, due to their relevance, they should be taken into account in a refined analysis.

A parametric study involving several 2D models with different values of the thickness of steel liner has been also performed. Results for a non-uniform annular gap, with maximum amplitude of $0.6 \mathrm{~mm}$, are shown in Figure 6. 


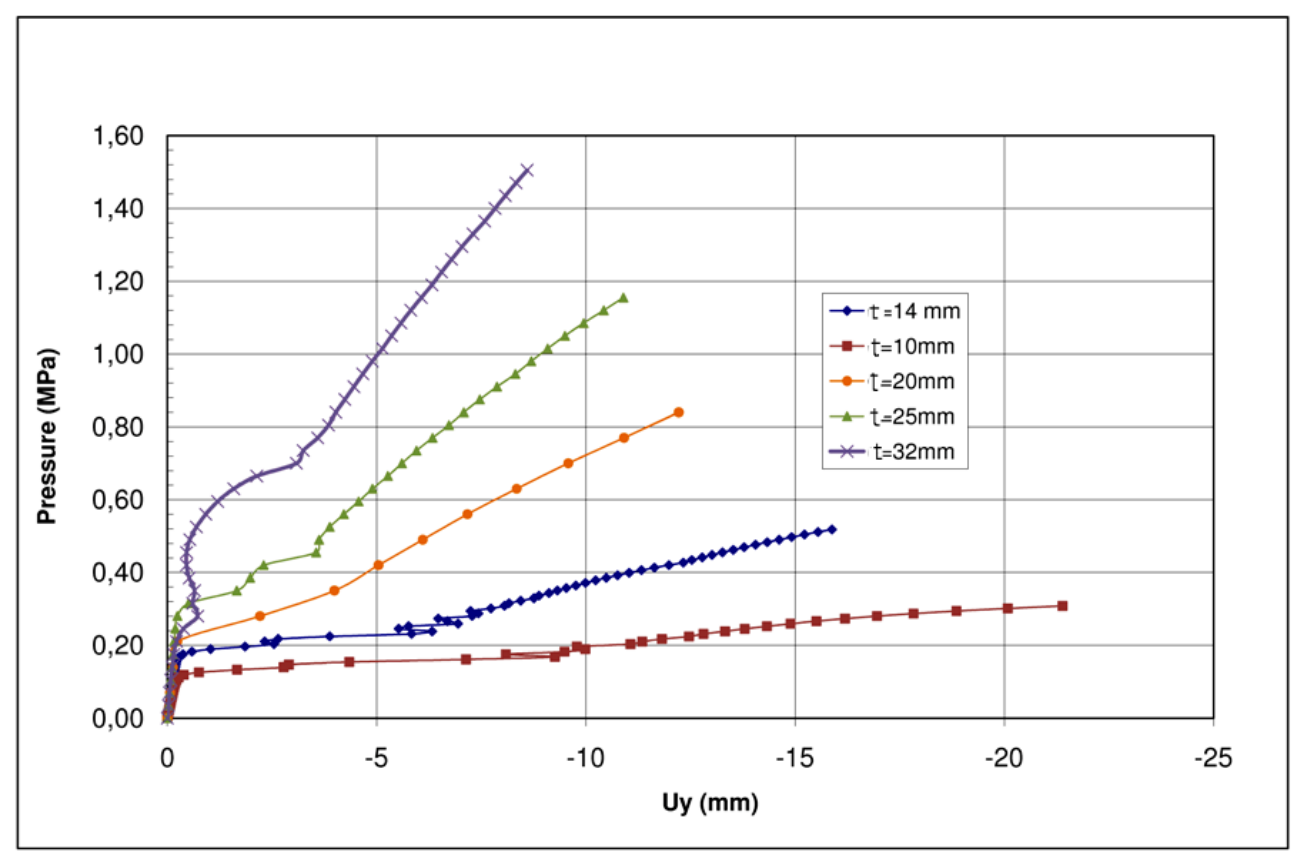

Figure 6. Pressure-displacement (radial displacement Uy) for different thickness of the liner. $(k=400 \mathrm{kN} / \mathrm{m})$

As shown in Figures 5 and 6 , there is a quasi-linear behavior (i.e., pseudo-linear branch) until the liner leans against the concrete pipeline. Then, as external pressure increases, so does the stiffness, until the ultimate strength is reached. Hence, the first yield point would represent the critical buckling load if the liner were not restricted (i.e., linear elastic buckling). However, this is an inelastic buckling case: the elastic yield point is reached $[11,21]$.

Results obtained for buckling pressure (Figure 7) agree with those of inelastic models (Montel and Jacobsen), and, as expected, yield lower values than elastic buckling models predicted by El Sawy et al [21]. 


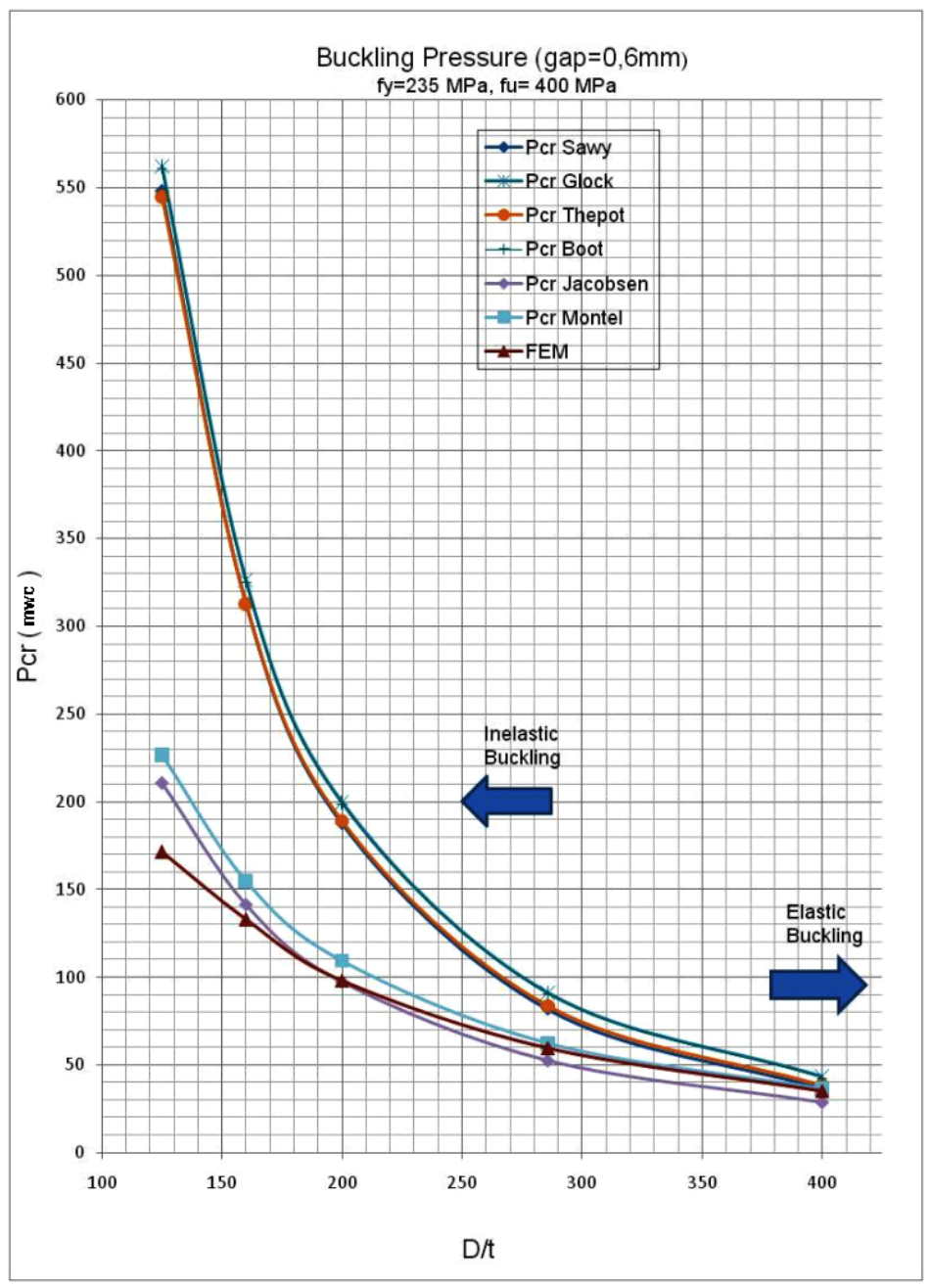

Figure 7. Results as compared with those from other approaches $(\mathrm{k}=400 \mathrm{kN} / \mathrm{m})$ expresed as meters of water colum (mwc)

\subsection{Eigenvalue buckling analysis of the 3D unconstrained steel liner}

Two types of assumptions are made in the models performed, i.e. either stiffened or unstiffened cylinders.

The not-encased steel liner is modeled as a 3D cylindrical shell with pinned support conditions at its end cross-sections. An elastic buckling analysis is performed. No geometric imperfections are considered. The first 6 buckling modes are calculated under uniform external pressure assumption.

Three different FE models and linear eigenvalue analyses are carried out and summarized:

- First model: an unstiffened 30 meter long liner.

- The second unstiffened liner model is anchored every $2.5 \mathrm{~m}$, so that nodal displacements are restrained at those cross-sections. Hence, this model consists of a 2.5 meter long unconfined cylinder. (Figure 8). 
Results obtained from these unstiffened cylinders are shown in Table 2.

- A stiffened 30 meter long liner model with a spacing of stiffeners of $2.5 \mathrm{~m}$ is analyzed.

Results are shown in Table 3 (Figure 9).

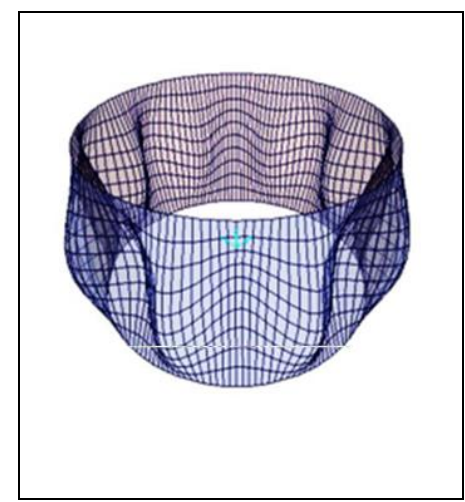

Figure 8. Bucked mode shape for a shell model with $L=2.500 \mathrm{~mm}$
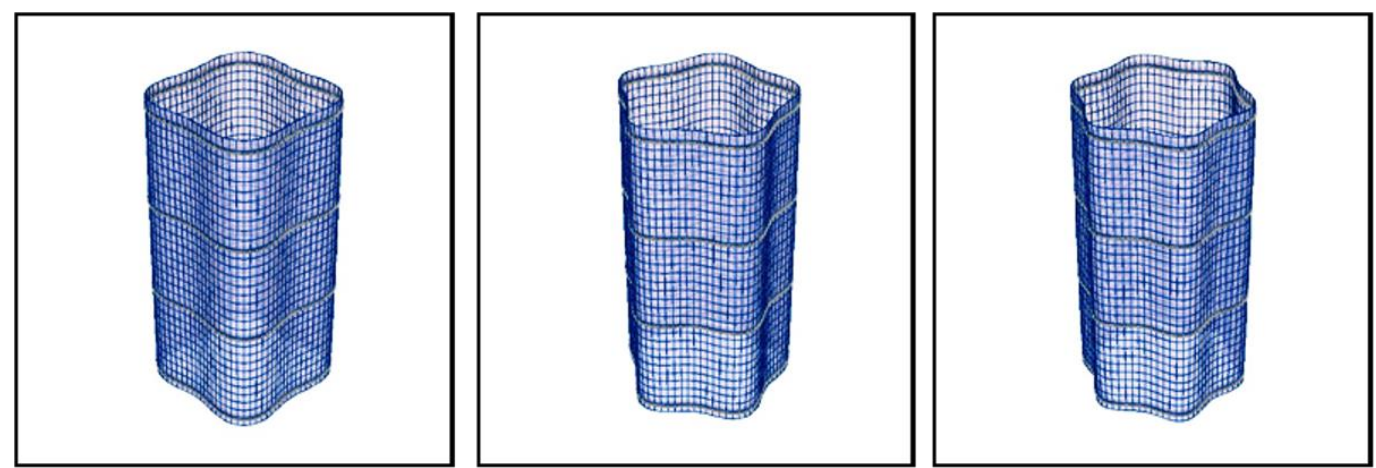

Figure 9. Buckling mode for multiple lobe buckling $n=4,5$ and 6 (stiffened cylinder case $L=30 \mathrm{~m}$ )

\begin{tabular}{|c|c|}
\hline Buckling mode (n) & Pcr \\
\hline $\mathrm{N}=4$ & $0.342 \mathrm{MPa}$ \\
\hline $\mathrm{N}=5$ & $0.400 \mathrm{MPa}$ \\
\hline $\mathrm{N}=6$ & $0.518 \mathrm{MPa}$ \\
\hline
\end{tabular}

Table 2. Critical load (MPa) for unstiffened cylinder eigenvalue buckling analysis 


\begin{tabular}{|c|c|c|c|c|c|}
\hline Case & Mode (n) & FEM & Roark & Donnel & Von Misses \\
\hline \multirow{3}{*}{$\mathrm{L}=30000$} & $\mathbf{4}$ & 0,111 & 0,050 & 0,111 & 0,100 \\
\cline { 2 - 6 } & $\mathbf{5}$ & 0,169 & 0,050 & 0,167 & 0,159 \\
\cline { 2 - 6 } & $\mathbf{6}$ & 0,244 & 0,050 & 0,238 & 0,231 \\
\hline & PCR & $\mathbf{0 , 1 1 1}$ & $\mathbf{0 , 0 5 0}$ & $\mathbf{0 , 1 1 1}$ & $\mathbf{0 , 1 0 0}$ \\
\hline
\end{tabular}

\begin{tabular}{|c|c|c|c|c|c|}
\hline Case & Mode (n) & FEM & Roark & Donnel & Von Misses \\
\hline \multirow{3}{*}{$\mathrm{L}=\mathbf{2 5 0 0}$} & $\mathbf{9}$ & 0,933 & 0,596 & 2,190 & 0,700 \\
\cline { 2 - 6 } & $\mathbf{1 0}$ & 0,940 & 0,596 & 1,617 & 0,783 \\
\cline { 2 - 6 } & $\mathbf{1 1}$ & 1,016 & 0,596 & 1,391 & 0,900 \\
\hline & PCR & $\mathbf{0 , 9 3 3}$ & $\mathbf{0 , 5 9 6}$ & $\mathbf{1 , 3 9 1}$ & $\mathbf{0 , 7 0 0}$ \\
\hline
\end{tabular}

Table 3. Critical load (MPa) for stiffened cylinder eigenvalue buckling analysis

\subsection{D nonlinear analysis of a constrained steel liner}

Two types of nonlinear 3D models have been implemented, i.e. an unstiffened liner and another with stiffeners. In both cases, a 25 meter long cylindrical steel liner is considered. Due to the existing symmetry, only a half of the cylinder was modeled. Annular gap amplitude of $0.6 \mathrm{~mm}$ and an ovality of $0.1 \mathrm{~mm}$ at the top of the cylinder are considered as initial imperfections. Both the annular gap and the deformability of the surrounding medium are accounted for by nonlinear linktype elements, whose stiffness is a function of the Young's modulus of the confinement and of $R$. A nonlinear direct-integration time-history analysis including large displacements and P-Delta effect is performed. A linearly step-by-step increase in the external pressure is applied.

The following parameters are chosen for P-Delta analysis:

- Substep size between 0.1 and 1e-4.

- Maximum constant-stiff iteration per step: 20.

- Maximum number of Newton-Raphson iterations per step: 20.

- Hilbert-Hughes-Taylor alpha (HHT) method, with $\alpha=-1 / 3$.

The unstiffened liner model consists of 2,400 shell-type elements and 2,706 gap-type elements. By linearly increasing the external pressure, a critical value of $0.53 \mathrm{MPa}$ is obtained, which corresponds to inelastic buckling i.e. the yield point is reached (Figure 10).

Results (pressure-deformation curves) from the 2D plane-strain model, as compared with those from the 3D model are shown in Figure 10. Hence, plane-strain assumption leads to conservative results: buckling pressure obtained from the full $3 \mathrm{D}$ model is $5 \%$ higher than that obtained with the plane-strain model. 


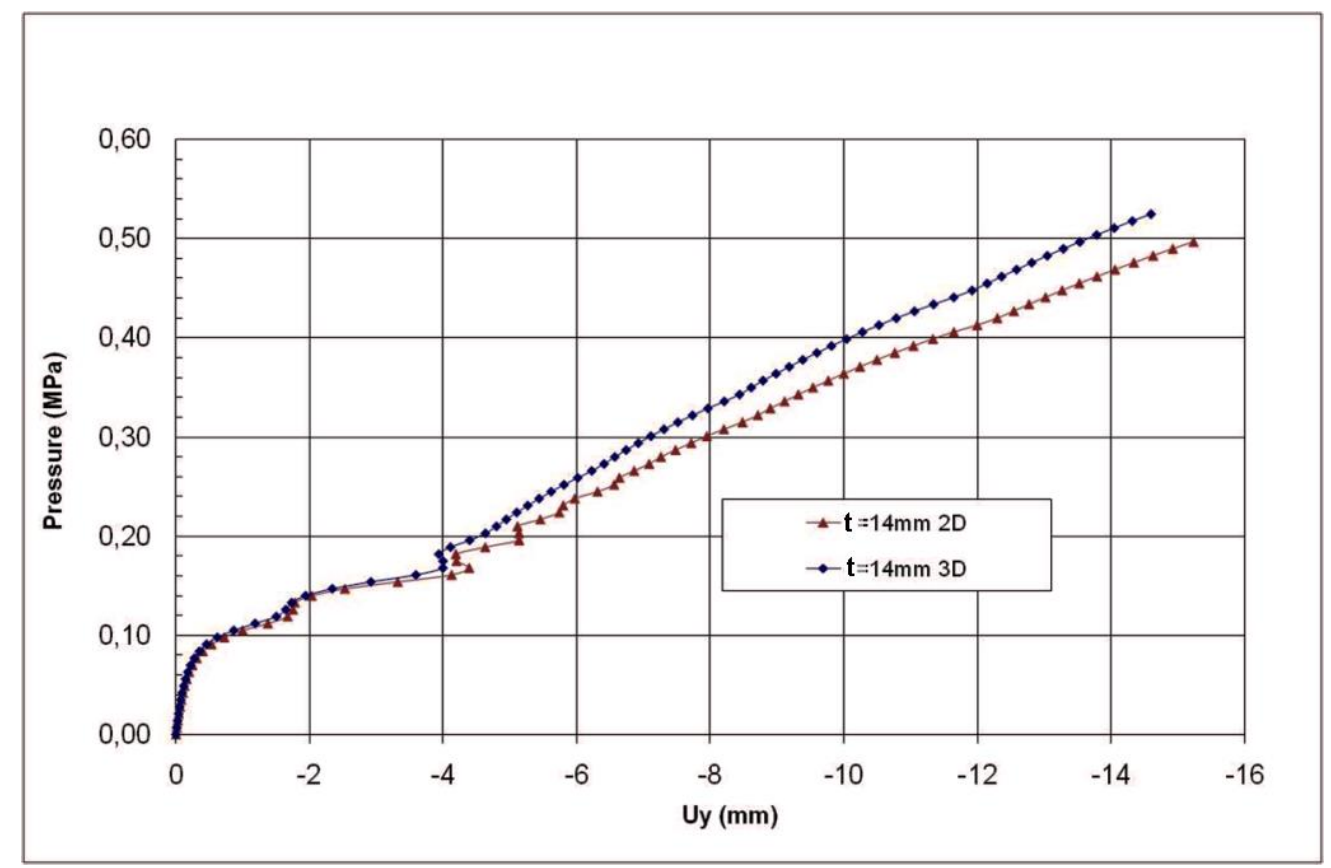

Figure 10. Comparison of results in 2D (plane deformation) and with a 3D model (full behavior). Uy (Radial Displacement)

On the other hand, another nonlinear 3D model including stiffeners has been performed.

Stiffeners restrain partially nodal displacements at the cross-section they belong to, so that the liner free-span is 2.5 meters. Stiffeners are modeled as beam-type elements with standard Lshaped $100.10 .10 \mathrm{~mm}$ cross-section.

The finite element model consists of 2,400 shell-type elements, 280 beam-type elements and 2,706 gap-type elements.

A critical pressure of $0.73 \mathrm{MPa}$ is obtained, which corresponds to inelastic buckling, i.e. the yield point is reached (Figure 12).

Buckling mode of the stiffened liner is shown in Figure 11. Failure corresponds to global instability.

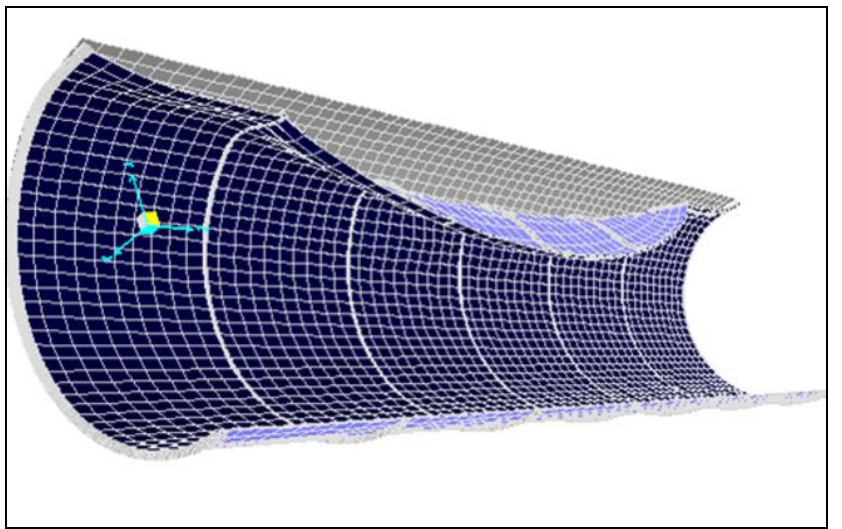

Figure 11. Buckling deformation of stiffened liner (single lobe) 
The pressure-displacement curve for a liner cross-section containing a stiffener is shown in Figure 12. As external pressure increases, the annular gap becomes zero at some points. Then, the system resistance is increased due to the confinement effect.

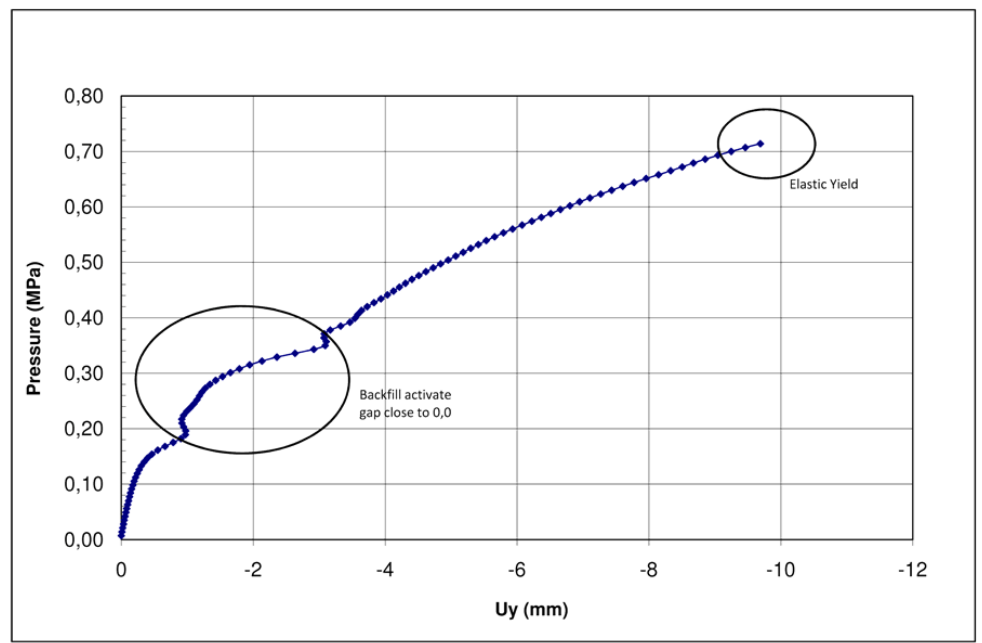

Figure 12. Pressure-Displacement (Radial Displacement Uy) curve for a constricted stiffened liner $D / t=285.7$

An alternative stiffened model has been implemented, in which stiffeners are fully constrained, in order to simulate their behavior as connectors. In this case, the liner fails by multiple-lobe buckling in the elastic range, with a critical pressure of $0.915 \mathrm{MPa}$.

As a whole, results from 3D model of stiffened steel liner in a pressure tunnel are adequate. However, the accuracy of a 2D model when including stiffeners is rather uncertain, mainly as regards the value of the effective width that should be considered in order to account for the stiffener effect.

For the case study (Figure $13 \mathrm{a}-\mathrm{b}$ ), the following results are outlined:

1. Critical pressure values obtained are $P_{c r}=0.94 \mathrm{MPa}$ for local buckling, $P_{c r}=0.915 \mathrm{MPa}$ (nonlinear buckling) for elastic local buckling, whereas $P_{c r}=0.73 \mathrm{MPa}$ for global buckling. Then, a safety factor between 1.34 and 1.04 is obtained.

2. If the steel liner is anchored at each 2.5 meters, the wall thickness $(14 \mathrm{~mm})$ is enough to prevent local buckling, i.e. multiple-lobe buckling mode may not occur.

3. Stiffeners provide enough reaction in order to constrain the cylinder and to transmit forces to the encasing concrete through friction (in other cases it may be mechanically). 
4. As external pressure exceeds the resistance of the welds between stiffeners and the shell, while friction between the stiffener and concrete pipeline is maintained, the shell may become detached from the stiffener.

5. Once this physical separation occurs, the free-span length increases from $2.5 \mathrm{~m}$ to $5 \mathrm{~m}$ and so on. Then, the critical buckling pressure may be reduced dramatically to that of a cylindrical unstiffened shell under external pressure $\left(P_{c r}=0.53 \mathrm{MPa}\right.$, with safety factor 0.76$)$. Hence, the steel liner may fail by global inelastic buckling.

6. The weakening of welds between the stiffeners and the shell, after decades of operation, may have contributed to the liner failure. (Figure 13)
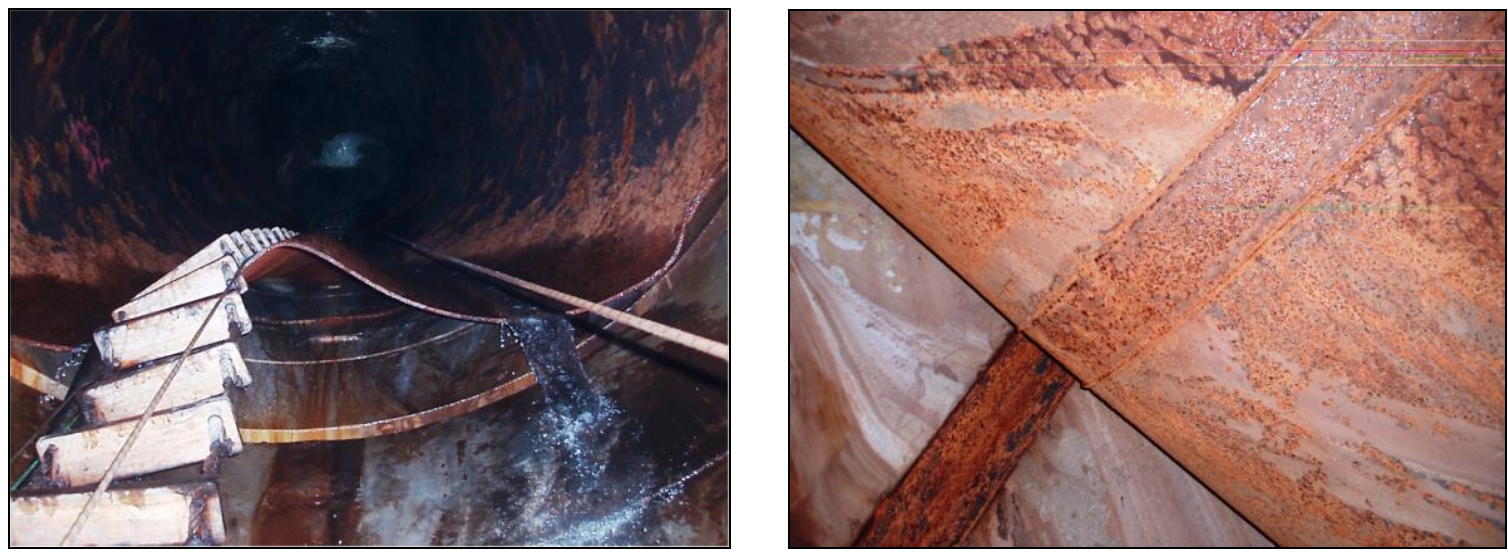

Figure 13. a-b. Views of damaged steel liner pressure tunnel

\section{Conclusions and recommendations}

Several conclusions and recommendations can be drawn from this study and are summarized in the following.

Appropriate methods and guidelines for buckling calculation of steel liners (either stiffened or unstiffened) are valuable tools for the safety assessment of these structures whose failure may lead to significant damages and financial loss. Also, those procedures must be helpful technical resources for the development of new hydroelectric projects, as well as for existing power plants. Some 2D nonlinear finite element models for assessing the buckling behavior of an unstiffened steel liner are presented. Also, 3D analyses (i.e., both eigenvalue buckling and nonlinear buckling analysis) of a 2.5 meter long liner-span between two consecutive stiffeners have been developed. Results drawn from both nonlinear $2 \mathrm{D}$ and $3 \mathrm{D}$ models agree quite well with other formulations. Plane-strain assumption (applied in 2D models) yields to conservative results as compared with 
those of 3D models, which give around 5\% greater value of critical pressure than those obtained with 2D models with plane-strain assumption.

The whole behavior of the concrete-encased thin-walled shell and stiffeners cannot be accurately simulated through 2D models. So, a full 3D nonlinear finite element model is advisable. Thus, the effect of the surrounding medium in which the liner is encased (i.e., rock and concrete rigidity, the annular gap, voids in the backfill,...) may be accounted for in more accurate models.

Yielding (inelastic buckling) seems to be a more frequent failure mode than buckling failure in actual hydroelectric steel liners, due to the usual values of thickness-to-diameter ratios $(100<D / t<300)$. Buckling failure may occur in the shell span between stiffeners (multiple-lobe buckling mode).

Finally, further research is needed in the following aspects:

- Characterization of practical limits (in terms of both geometric and material parameters) involved in concrete-encased steel liner modeling and analysis, particularly with regard to differentiating between buckling collapse and failure due to yielding (elastic and inelastic buckling).

- The behavior of high-strength steel liners at high stress rates.

- Design methods and guidelines for analysis of stiffened steel liners. No conclusive studies in this field have been found. Hence, some parametric 3D finite element models for simulating the global behavior of both the cylindrical shell and stiffeners could be of interest. The most important parameters may be: the moment of inertia of stiffeners, the ratio of spacing of stiffeners to diameter, the thickness to diameter ratio and yield limit, among others.

- The inclusion of imperfections, particularly those due to of welding and manufacturing, which may have relevant effect on elastic buckling.

\section{Acknowledgements}

The writers would like to express their thanks to María Angeles Cervera Iranzo and Jorge García Cervera for their support and understanding. 


\section{References}

[1] F.E. Hachen, J. Schleiss, The design of steel-lined pressure tunnels and shafts, The International Journal on Hydropower \& Dams. 16(3) (2009) 142-151.

[2] J. Amdahl, Buckling and Ultimate Strength of Marine Structures, Chapter 5, Buckling of cylindrical Shells. TMR4205, MTS-2010.01.11, (2010).

[3] Det Norske Veritas (DNV), Recommended Practice DNV RPC201: Buckling strength of plated structures, (2002).

[4] ECCS, Buckling of steel shells, European Recommendations, Technical Committee No. 8. 1988.

[5] ECCS, European recommendations for steel construction buckling of shells, Technical committee No.8. 1984.

[6] J.G. Teng, J.M. Rotter, Buckling of thin metal shells, Spon Press. (2004) 159-174.

[7] S.P. Timoshenko, J. Gere, Theory of elastic stability, McGraw-Hill, (1963).

[8] C.M. Wang, C.Y. Wang, J.N. Reddy, Exact solutions for buckling of structural members, CRC Press, (2005).

[9] Z.P. Bazant, L. Cedolin, Stability of Structures. Elastic, Inelastic, Fracture and Damage Theories, Dover Publications, (2003).

[10] M. Omara L.K. Guice, W.T. Straughan, F.A. Akl, Buckling Models of Thin Circular Pipes Encased in Rigid Cavity, J. Eng. Mech.123 (1997) 1294-1301.

[11] D. Vasilikis, S.A. Karamanos, Stability of confined thin-walled steel cylinders under external pressure, International Journal of Mechanical Sciences. 52 (2009) 21-32.

[12] L.H. Donnell, "Effect of Imperfections on Buckling of Thin Cylinders Under External Pressure" J. Applied Mech. Vol 23, No 4 (1956).pp 569

[13] E.W. Vaughan. "Steel linings for pressure shafts in solid rock. ASCE Journal of the Power Division, 82. (1956). 949-989.

[14] M. Borot. “Essais des Conduits Metálliques Noyées Dans du Béton”. La Houille Blanche (1976). 881-888

[15] U.S. Army Corps of Engineers, Engineering and Design. Tunnels and shafts in rock. EM 1110 2-2901, (1997).

[16] D. Glock. “Überkritisches Verhalten eines Starr Ummantelten Kreisrohres bei Wasserdrunck von Aussen und Temperaturdehnung" (Post-critical behavior of a rigidly encased circular pipe subject to external water pressure and thermal extension). Der Stahlbau No 7(1977) 212-219.

[17] S. Jacobsen, Buckling of circular rings and cylindrical tubes under external pressure, Water Power \& Dam Construction. 26 (1974) 400-407. 
[18] S. Jacobsen, Pressure distribution in steel line rock tunnels and shafts, Water Power \& Dam Construction. 29 (1977) 47-51.

[19] J.C. Boot, Elastic buckling of cylindrical pipe linings with small imperfections subject to external pressure, Trenchless Technol. Res. 12 (1-2) (1998) 3-15.

[20] K.M. El-Sawy, Inelastic Stability of Loosely Fitted Cylindrical Liners, J. Struct. Eng. 128 (7) (2002) 934941.

[21] K.M. El Sawy, M.I. Sweedan, Elastic Stability Analisys of loosely fitted thin liners. A proposed simplified procedure and evaluation of existing solutions, Tunneling and Underground Space Technology. 25 (2010) 689-701.

[22] O. Thépot. "A new design method for non-circular sewer linings. Trenchless Technology Research 15 No. 1. (2000) $25-41$

[23] D. Berti, R. Stutzman, E. Lindquist, M. Eshghipour, Buckling of steel tunnel liner under external pressure, J. Energy Eng. 124(3) (1998) 55-89.

[24] Z. Li, L. Wang, Z. Guo, H. Shu, Elastic buckling of cylindrical pipe linings with variable thickness encased in rigid host pipes, Thin-Walled Structures. 51(2012) 10-19

[25] D.F. Windenburg, C. Trilling. "Collapse by Instability of Thin Cylindrical Shells Under External Pressure". Transactions. ASMA (1934).

[26] M. Pircher, P.A. Berry, X. Ding and R.Q. Bridge, The shape of circumferential weld-induced imperfections in thin-walled steel silos and tanks, Thin-Walled Structures. 39 (2001) 999-114.

[27] CSI, CSI Analysis Reference Manual for SAP 2000, ETABS and SAFE. 2009.

[28] R. Montel. " Formule Semi-empirique pour la Détermination de la Pression Extérieure Limite d'inestabilité des Conduits métalliques Lisses Noyées Dans du Béton". La Houille Blanche (1960) 560-568. [29] B.N. Prabu, R. Srinivasan, K.A.S. Naarayen, Finite Element Analysis of Buckling of Thin Cylindrical Shell Subjected to Uniform External Pressure, Journal of Solid Mechanics. 1(2) (2009) 148-158.

[30] C. Lopez Jimeno, F. Mendaña Saavedra, Ingeo Túneles, Serie: Ingeniería de túneles. Libro 1. Ed. Carlos Lopez Jimeno. 1.998. pp 367-368. 


\section{CAPTIONS}

Figure 3. Four-node finite shell element

Figure 4. Gap contact-type element

Figure 3. View of damaged steel liner pressure tunnel

Figure 4. Hydroelectric pressure tunnel longitudinal profile of case study

Figure 5. Pressure-displacement (radial displacement Uy in the node located at the top of the cylinder, where geometrical imperfection is applied) for different stiffness values of the backfill $(k)$. D/t=285.7

Figure 6. Pressure-displacement (Radial Displacement Uy) for different thickness of the liner

Figure 7. Results as compared with those from other approaches

Figure 8. Bucked mode shape for a shell model with $L=2.500 \mathrm{~mm}$

Figure 9. Buckling mode for multiple lobe buckling $n=4,5$ and 6 (stiffened cylinder case $L=30 \mathrm{~m}$ )

Figure 10. Comparison of results in 2D (plane deformation) and with a 3D model (full behavior). Uy (Radial Displacement)

Figure 11. Buckling deformation of stiffened liner (single lobe)

Figure 12. Pressure-Displacement (Radial Displacement Uy) curve for a constricted stiffened liner. $D / t=285.7$

Figure 13. a-b. Views of damaged steel liner pressure tunnel

Table 1. Appropriate calculation methods depending on the type of pressure tunnel.

Table 2. Critical load (MPa) for unstiffened cylinder eigenvalue buckling analysis

Table 3. Critical load (MPa) for stiffened cylinder eigenvalue buckling analysis 